\title{
MODELLING THE SPECTRAL ENERGY DISTRIBUTIONS OF AGB STARS IN THE LMC
}

\author{
M. A. T. GROENEWEGEN \\ Max-Planck-Institut für Astrophysik, Garching, Germany \\ J. TH. VAN LOON \\ European Southern Observatory, Garching, Germany \\ P. A. WHITELOCK \\ South African Astronomical Observatory \\ Observatory, South Africa \\ P. R. WOOD \\ Mount Stromlo and Siding Spring Observatories \\ Canberra ACT, Australia
}

AND

A. A. ZIJLSTRA

European Southern Observatory, Garching, Germany

\begin{abstract}
The spectral energy distributions of 16 AGB stars in the LMC are fitted. The 6 known oxygen-rich stars are well fitted with silicate dust. Due to the completely different absorption properties of silicate and carbonaceous dust we argue that of the 10 stars of unknown spectral type, 1 is probably O-rich while the other 9 are probably C-rich.

The Period-Luminosity relation is discussed. Both the O- and C-rich LPVs in this study appear to lie on extensions of $P-L$ relations derived for stars with much shorter periods.
\end{abstract}

\section{Introduction}

Modelling the spectral energy distributions (SEDs) of late-type stars provides information on their mass-loss rates and luminosities. Essentially all model fitting of this kind up to now has been done on AGB stars in our Galaxy (e.g. Groenewegen 1995 on carbon stars; Justtanont \& Tielens 1992 on oxygen-rich stars). 
The advantage of doing similar modelling for AGB stars in extra-galactic systems is that the distance is known (which is not usually the case for AGB stars in the Galaxy), and that one can then estimate the dependence of mass loss on metallicity.

The requirement to get reliable results from the model fitting is primarily a sufficiently large data set of photometric observations covering a broad range in wavelength. For AGB stars in our Galaxy these data either exist in the literature or are relatively easily obtained. In contrast, observations of AGB stars in the Magellanic Clouds, in particular in the near-infrared where most AGB stars emit most of their energy, have started to be performed only recently (Wood et al. 1992, Zijlstra et al. 1996).

In this paper we present the first results on the modelling of 16 AGB stars in the LMC, from which luminosities are derived. We also discuss the Period-Luminosity relation. A paper is in preparation which will include a discussion of the mass-loss rates and possible evolutionary consequences.

\section{The Sample}

From the available literature we selected all 16 AGB stars in the LMC which were detected by the IRAS satellite and for which pulsation periods have been determined. The periods and near-infrared data are taken from Wood et al. (1992), Zijlstra et al. (1996, 1998) and Wood (1998). One star (TRM 60) was previously fitted in Groenewegen et al. (1995), as one of only two AGB stars outside our Galaxy for which a silicate feature has been detected up to now. The near-infrared observations were supplemented by IRAS data. In order to obtain the best possible IRAS 12 and $25 \mu$ m fluxes we inspected two-dimensional images and one-dimensional co-adds of IRAS scans using the GIPSY software package (Assendorp et al. 1995). No useful 60 or $100 \mu \mathrm{m}$ data could be obtained because of the high and variable background emission in the direction of the LMC.

The periods range between 1040 and 1400 days for the stars we believe to be O-rich (see below), and between 515 and 900 days for the stars we believe to be C-rich. These periods are significantly longer than those of LPVs selected from optical surveys (see Sect. 4). This is related to the fact that the stars were selected from the IRAS data-base which favors high mass-loss rates and high luminosities.

Six of the 16 stars are known oxygen-rich stars, either from the detection of $\mathrm{OH}$ maser emission (Wood et al. 1992) or the silicate dust feature (Groenewegen et al. 1995; TRM 60), or from optical spectroscopy (van Loon et al. 1998). The chemical type of the other stars is unknown, but we will argue later that the sample can be separated into O- and C-rich based on the modelling of the SEDs. 


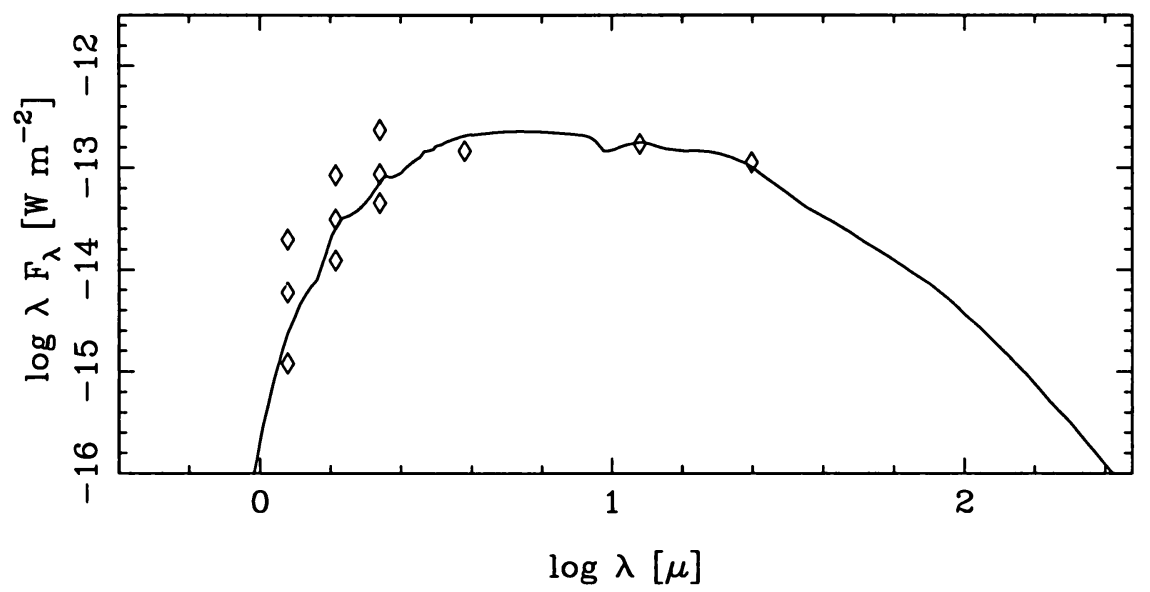

Figure 1. Fit to the spectral energy distribution of the oxygen-rich AGB star LMC 1506. Plotted are the IRAS data points at 12 and $25 \mu \mathrm{m}$ and near-infrared data. The 3 values plotted for a given wavelength represent the fluxes at maximum, mean and minimum light.

\section{Results}

The SEDs are modelled with the radiative transfer model of Groenewegen (1993). Figure 1 shows the model fit to one of the known oxygen-rich stars. The dust opacity for silicates (Volk \& Kwok 1988) is used. This type of dust gave the best results in fitting the $8-13 \mu \mathrm{m}$ spectra of two AGB stars in the LMC and SMC (Groenewegen et al. 1995). The stellar photosphere is represented by an M5 model atmosphere (Fluks et al. 1994). This is the reason the model fit is not smooth in the NIR. The fitting is a twostep process. First the optical depth is determined by fitting the IRAS $S_{12} / S_{25}$ flux ratio. Then the final values of the mass-loss rate and luminosity are derived by adjusting the model to the observed $12 \mu \mathrm{m}$ flux-density. Therefore the model predicts the near-infrared photometry and is not fitted to it. As the star is known to be oxygen-rich the model should lie between the observed fluxes at minimum and maximum light, as is indeed the case. In fact, all 6 known oxygen-rich stars are well fitted with silicate dust.

Figure 2 shows model fits to an AGB star of unknown spectral type. The upper panel is a fit to the IRAS data using silicate dust. This model clearly does not fit the observations. The lower panel is a fit using dust composed of $95 \%$ amorphous carbon (Rouleau \& Martin 1991) with 5\% silicon carbide (Pégourie 1988) and the stellar photosphere represented by a $2500 \mathrm{~K}$ blackbody. It is clear that the model with carbon-rich dust fits the data reasonably well, while the model with silicate dust completely fails. This is due to the completely different absorption properties of silicate and carbonaceous dust. We therefore argue that this star is a carbon star. Using 

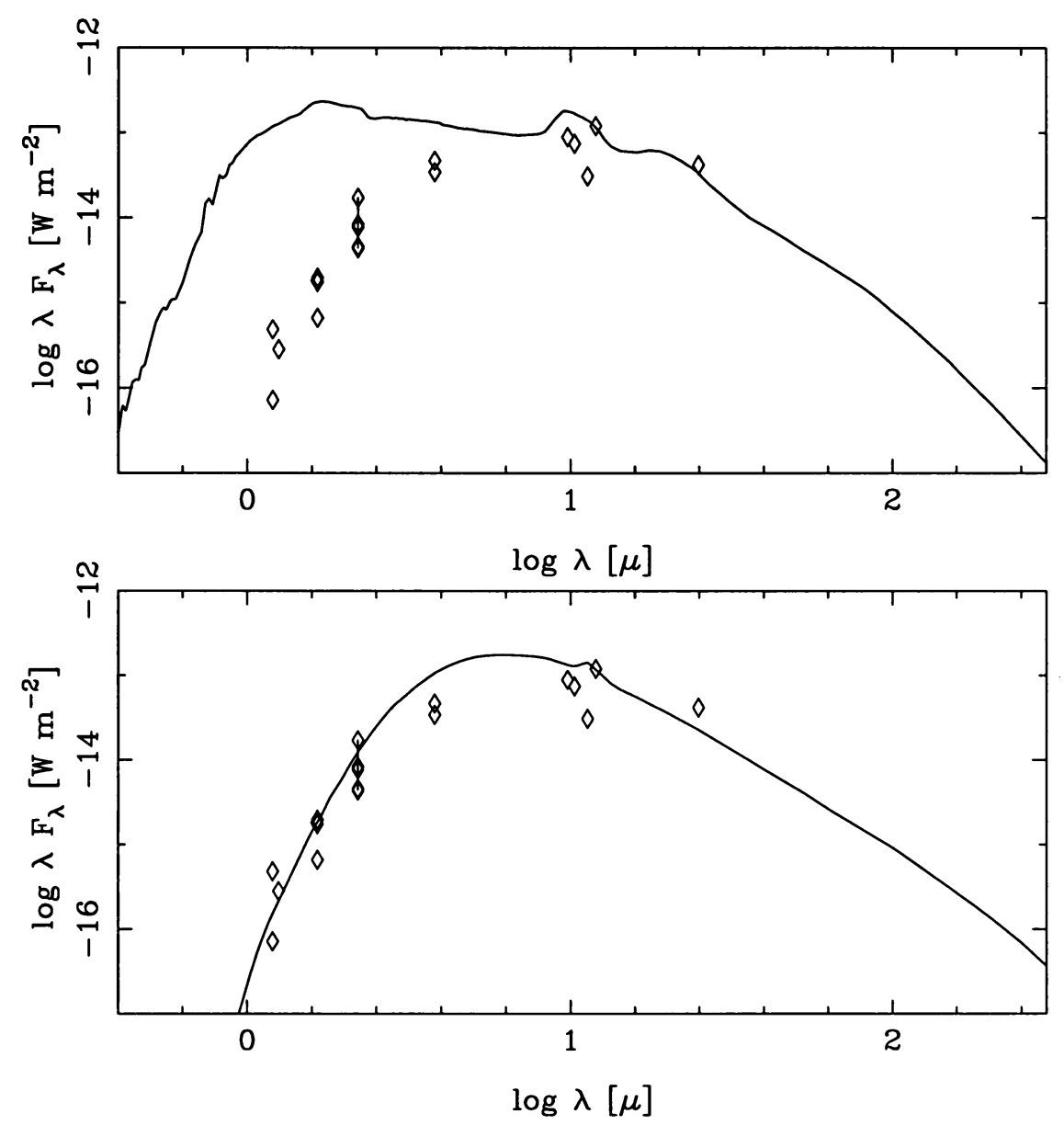

Figure 2. Fit to the spectral energy distribution of LMC 570, of unknown spectral type. In the upper panel silicate dust is used, in the lower panel amorphous carbon mixed with $5 \%$ silicon carbide.

this approach we find that of the 10 stars of unknown spectral type, 1 is probably O-rich, while the other 9 are probably C-rich.

\section{Period-Luminosity Relations}

The Period-Luminosity relation $(P-L$ relation) is a useful empirical tool. $P-L$ relations for $\mathrm{O}-$ and $\mathrm{C}$-rich stars in the LMC were derived by Feast et al. (1989). Recently, an improved relation for C stars was presented by Groenewegen \& Whitelock (1996). Both relations were limited to stars with periods less than $\sim 400-500$ days because they were based on optically visible stars. The stars in the current sample have much longer periods compared to previous samples. It includes stars with periods of 700-1400 days compared to $\lesssim 500$ days in the earlier LMC samples (Zijlstra et al. 1996; 

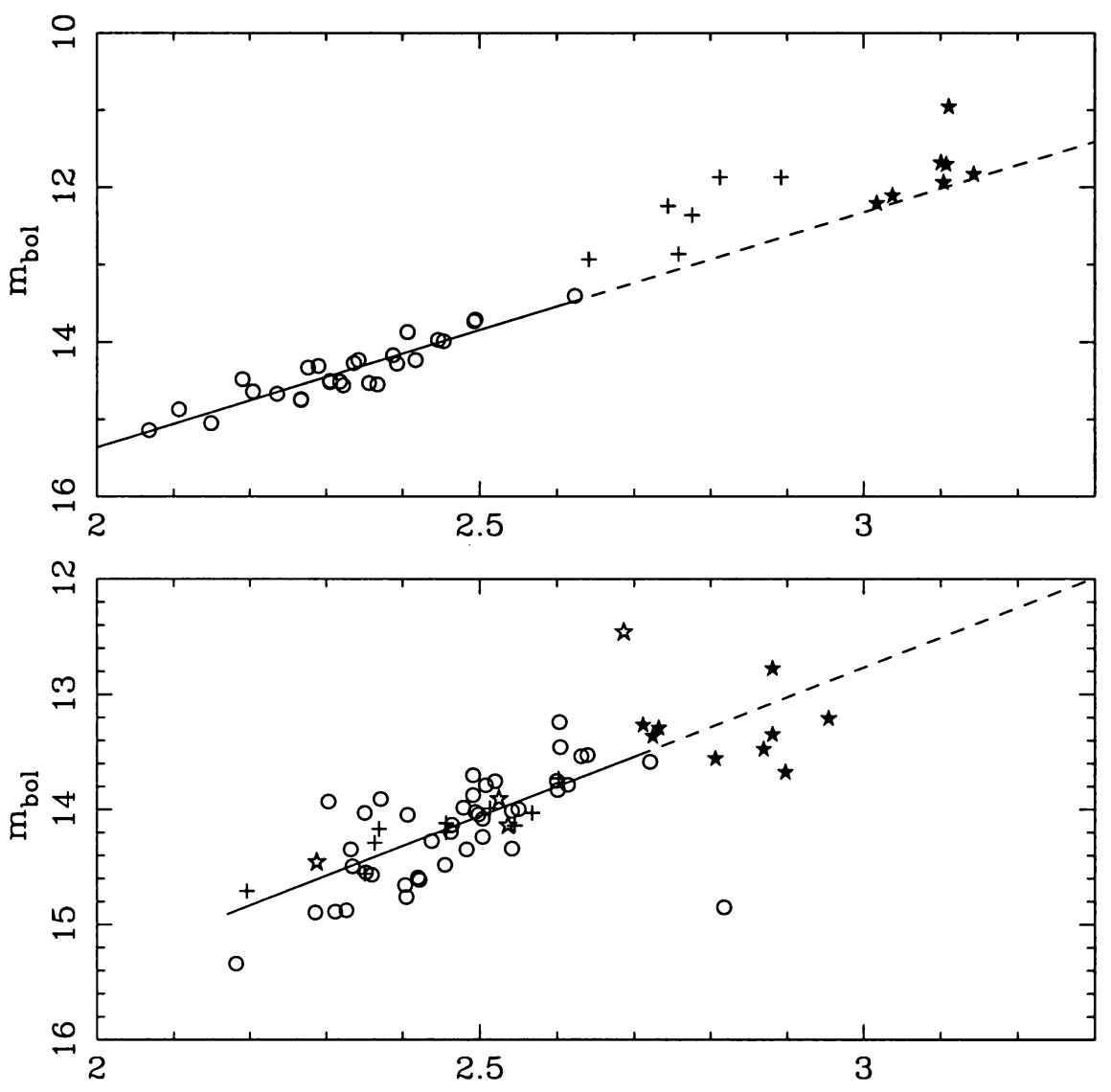

$\log P$

Figure 3. Period-Luminosity relation for O-rich (upper panel) and C-rich (lower panel) AGB stars in the LMC. The solid and dashed lines are least-squares fits (and the extrapolations, respectively) to the data points represented by the open symbols in the upper panel and open symbols and crosses in the lower one (except the two obvious outliers). The filled symbols represent the stars from the present sample. See text for further information.

Wood et al. 1992). The stars in the present study have better-determined luminosities and the separation between $\mathrm{O}$ - and C-rich is made more effectively than was previously possible.

In Fig. 3 the $P-L$ relations are shown. In the top panel the data used by Feast et al. (1989) for O-rich stars are shown (open circles and crosses). The solid line represents the $P-L$ relation derived by Feast et al. from a least-squares fit to the open circles; the dashed line is its extrapolation to longer periods. The filled symbols are data from the present paper. These points lie on the extrapolation of the Feast et al. $P-L$ relation derived for O-rich Miras with periods < 420 days. 
In the lower panel the data from Groenewegen \& Whitelock (1996) on $\mathrm{C}$ stars in the LMC are plotted, together with their $P-L$ relation and its extrapolation. This $P-L$ relation was derived from the crosses and open symbols which represent stars with periods less than 520 days. The $\mathrm{C}$ stars from the present sample (filled symbols) lie on the extrapolated $P-L$ relation.

In conclusion, the study of IRAS-detected AGB stars in the LMC allows one to investigate the most luminous AGB stars with high mass-loss rates and long pulsation periods. The model fitting enables a reliable determination of the carbon- or oxygen-rich nature of a star (due to the completely different absorption properties of silicate and carbonaceous dust). Both the $\mathrm{O}$ - and C-rich LPVs in this study appear to lie on extensions of $P-L$ relations derived for stars with much shorter periods.

\section{References}

Assendorp, R., Bontekoe, T. R., de Jonge, A. R. W., Kester, D. J. M., Roelfsema, P. R. \& Wesselius, P. R. 1995, AछA Supp., 110, 395

Feast, M. W., Glass, I. S., Whitelock, P. A. \& Catchpole, R. M. 1989, MNRAS, 241, 375

Fluks, M. A., Plez, B., Thé, P.S., de Winter, D., Westerlund, B.E. \& Steenman, H. C. 1994, A\&A Supp., 105, 311

Groenewegen, M. A. T. 1993, Ph.D. Thesis, University of Amsterdam, Chapter 5

Groenewegen, M. A. T. 1995, A\&A, 293, 463

Groenewegen, M. A. T., Smith, C. H., Wood, P. R., Omont, A. \& Fujiyoshi, T. 1995, ApJ, 449, L119

Groenewegen, M. A. T. \& Whitelock, P. A. 1996, MNRAS, 281, 1347

Justtanont, K. \& Tielens, A. G. G. M. 1992, $A p J, 389,400$

Pégourié, B. 1988, $A \& A, 194,335$

Rouleau, F. \& Martin, P. G. 1991, ApJ, 377, 526

van Loon, J. Th., Zijlstra, A. A., Whitelock, P. A., te Lintel Hekkert, P., Chapman, J. M., Loup, C., Groenewegen, M. A. T., Waters, L. B.F.M. \& Trams, N.R. 1998, $A \& A$, 329,169

Volk, K. \& Kwok, S. 1988, ApJ, 331, 435

Wood, P. R., Whiteoak, J. B., Hughes, S. M. G., Bessell, M.S., Gardner, F. F. \& Hyland, A.R. $1992, A p J, 397,552$

Wood, P. R. 1998, submitted

Zijlstra, A.A., Loup, C., Waters, L.B.F.M., Whitelock, P.A., van Loon, J.Th. \& Guglielmo, F. 1996, MNRAS, 279, 32

Zijlstra, A. A. et al. 1998, in preparation

\section{Discussion}

Joyce: I have completed a photometric monitoring program of long-period variables with dense circumstellar shells similar to that of Whitelock, but with a more extreme selection criterion, $K-[11 \mu \mathrm{m}]>4$. I found one Ctype star, AFGL 190, with $P=1050 \mathrm{~d}$. This star is sufficiently faint and red $(K \approx 14 ; L \approx 7)$ that an analog in the LMC may not be detectable in the near-infrared. 